\title{
Prognostic significance of performing universal HER2 testing in cases of advanced gastric cancer
}

\author{
Paula Jiménez-Fonseca $^{1}$ - Alberto Carmona-Bayonas ${ }^{2} \cdot$ Maria Luisa Sánchez Lorenzo $^{1} \cdot$ \\ Javier Gallego Plazas ${ }^{3} \cdot$ Ana Custodio $^{4} \cdot$ Raquel Hernández $^{5}$. \\ Marcelo Garrido $^{6}$ - Teresa García $^{2}$ - Isabel Echavarría ${ }^{7}$. Juana María Cano ${ }^{8}$. \\ Alberto Rodríguez Palomo ${ }^{9}$ Monserrat Mangas $^{10} \cdot$ Ismael Macías Declara $^{11}$ • \\ Avinash Ramchandani ${ }^{12}$ - Laura Visa ${ }^{13}$. Antonio Viudez ${ }^{14}$. \\ Elvira Buxó $^{15}$ - Asunción Díaz-Serrano ${ }^{16}$ - Carlos López ${ }^{17}$ • Aitor Azkarate ${ }^{18}$. \\ Federico Longo ${ }^{19} \cdot$ Eduardo Castañón $^{20} \cdot$ Rodrigo Sánchez Bayona ${ }^{20}$. \\ Paola Pimentel $^{21}$ - Maria Luisa Limón ${ }^{22}$ - Paula Cerdá 23 - Renata Álvarez Llosa ${ }^{24}$. \\ Raquel Serrano $^{25} \cdot$ Maria Pilar Felices Lobera ${ }^{26}$ - María Alsina ${ }^{27}$. \\ Alicia Hurtado Nuño ${ }^{28}$ Carlos Gómez-Martin ${ }^{16}$
}

Received: 23 May 2016/Accepted: 26 August 2016/Published online: 6 September 2016

(c) The International Gastric Cancer Association and The Japanese Gastric Cancer Association 2016

\begin{abstract}
Background Trastuzumab significantly improves overall survival (OS) when added to cisplatin and fluoropyrimidine as a treatment for HER2-positive advanced gastric cancers (AGC). The aim of this study was to evaluate the impact of
\end{abstract}

Electronic supplementary material The online version of this article (doi:10.1007/s10120-016-0639-8) contains supplementary material, which is available to authorized users.

Paula Jiménez-Fonseca

palucaji@hotmail.com

1 Medical Oncology Department, Hospital Universitario Central de Asturias, Avenida de Roma s/n, 33011 Oviedo, Spain

2 Hematology and Medical Oncology Department, Hospital Universitario Morales Meseguer, UMU, IMIB, Murcia, Spain

3 Medical Oncology Department, Hospital General Universitario de Elche, Alicante, Spain

4 Medical Oncology Department, Hospital Universitario La Paz, Madrid, Spain

5 Medical Oncology Department, Hospital Universitario de Canarias, Santa Cruz De Tenerife, Spain

6 Medical Oncology Department, Pontificia Universidad Católica de Chile, Santiago De Chile, Chile

7 Medical Oncology Department, Hospital Universitario Gregorio Marañón, Madrid, Spain

8 Medical Oncology Department, Hospital General de Ciudad Real, Ciudad Real, Spain the gradual implementation of HER2 testing on patient prognosis in a national registry of AGC.

Methods This Spanish National Cancer Registry includes cases who were consecutively recruited at 28 centers from January 2008 to January 2016. The effect of missing HER2 status was assessed using stratified Cox proportional hazards $(\mathrm{PH})$ regression.

Results The rate of HER2 testing increased steadily over time, from $58.3 \%$ in 2008 to $92.9 \%$ in 2016. HER2 was

9 Pharmacy Department, Hospital Universitario Central de Asturias, Oviedo, Spain

10 Medical Oncology Department, Hospital Galdakao-Usansolo, Usansolo, Spain

11 Medical Oncology Department, Hospital Universitario Parc Tauli, Sabadell, Spain

12 Medical Oncology Department, Hospital Universitario Insular de Gran Canaria, Las Palmas De Gran Canaria, Spain

13 Medical Oncology Department, Hospital Universitario del Mar, Barcelona, Spain

14 Medical Oncology Department, Complejo Hospitalario de Navarra, Pamplona, Spain

15 Medical Oncology Department, Hospital Clínic, Barcelona, Spain

16 Medical Oncology Department, Hospital Universitario Doce de Octubre, Madrid, Spain

17 Medical Oncology Department, Hospital Universitario Marqués de Valdecilla, Santander, Spain

18 Medical Oncology Department, Hospital Universitario Son Espases, Mallorca, Spain 
positive in 194 tumors $(21.3 \%)$. In the stratified Cox PH regression, each $1 \%$ increase in patients who were not tested for HER2 at the institutions was associated with an approximately $0.3 \%$ increase in the risk of death: hazard ratio, 1.0035 (CI $95 \%, 1.001-1.005), P=0.0019$. Median OS was significantly lower at institutions with the highest proportions of patients who were not tested for HER2.

Conclusion Patients treated at centers that took longer to implement HER2 testing exhibited worse clinical outcomes. The speed of implementation behaves as a qualityof-care indicator. Reviewed guidelines on HER2 testing should be used to achieve this goal in a timely manner.

Keywords Gastric cancer - HER2 testing - Trastuzumab . Personalized medicine - Quality of care

\section{Introduction}

Advanced gastric cancers (AGC) display several chromosomal and genetic abnormalities. Notably, up to $37 \%$ of AGCs show amplification of the genes related to receptor tyrosine kinase/Ras signaling pathways [1, 2]; this has been corroborated by the recently proposed molecular classification by The Cancer Genome Atlas [3]. One major challenge is therefore to implement strategies that select patients according to the intrinsic molecular characteristics of their cancer so that targeted agents can be optimized to improve overall survival (OS).

In 1986, some gastric cancers were found to exhibit amplified human epidermal growth factor receptor 2

19 Medical Oncology Department, Hospital Universitario Ramón y Cajal, Madrid, Spain

20 Medical Oncology Department, Clínica Universidad de Navarra, Pamplona, Spain

21 Medical Oncology Department, Hospital Santa Lucía, Cartagena, Spain

22 Medical Oncology Department, Hospital Universitario Virgen del Rocío, Seville, Spain

23 Medical Oncology Department, Instituto Oncológico Teknon, Barcelona, Spain

24 Medical Oncology Department, Complejo Hospitalario de Orense, Ourense, Spain

25 Medical Oncology Department, Hospital Universitario Reina Sofía de Córdoba, Córdoba, Spain

26 Medical Oncology Department, Hospital Universitario Miguel Servet de Zaragoza, Zaragoza, Spain

27 Medical Oncology Department, Hospital Universitario Vall d'Hebron and VHIO_-Vall d'Hebron Institute of Oncology, Barcelona, Spain

28 Medical Oncology Department, Hospital Universitario Fundación Alcorcon, Madrid, Spain
(HER2) [4]. In the following decade, it was estimated that between 9 and $36 \%$ of all tumors overexpress or amplify HER2 depending on location and histopathological subtype [5-7]. Initial clinical trials indicated that trastuzumab, an anti-HER2-targeted agent, possesses considerable activity against HER2-positive AGC [8, 9].

The ToGa phase III trial evaluated HER2 amplification using fluorescence in situ hybridization (FISH) and HER2 overexpression by immunohistochemistry (IHC) in 3807 patients with AGC or adenocarcinoma of the gastroesophageal junction. The results established a clear benefit of trastuzumab in terms of OS [hazard ratio (HR) 0.74 , $P=0.0046]$. The subgroup with HER2 expression of IHC3 + or IHC2+/FISH+ obtained the greatest survival benefit (16 vs. 11.8 months) [10]. Trastuzumab not only improves OS but also helps to preserve patient quality of life [11]. Thus, the introduction of trastuzumab into the frontline treatment of HER2-positive AGC ushered in the age of targeted therapy and personalized medicine for this pathology.

Based on these data, the European Medicines Agency approved trastuzumab in combination with chemotherapy to treat HER2-positive AGC in 2010. Since then, HER2 testing has become a standard practice. However, research has paid less attention to the process of implementing HER2 determination across centers and the main barriers to its use. Novel medical technologies such as HER2 testing are gradually implemented after a certain period of time, and the speed at which this process is completed (e.g., the time taken to change the standard of care) may impact clinical outcomes in a complex manner. As such, the introduction of systematic HER2 testing could be an attractive model to exemplify the effect of predictive biomarkers for response to targeted treatments in the community. Therefore, the aim of the study reported in the present paper was to evaluate how changes in the proportions of patients with AGC who were not tested for HER2 at institutions influenced the OS of those patients during the period from 2008 to 2016.

\section{Patients and methods}

\section{Patients}

This Spanish National Cancer Registry includes cases who were consecutively recruited at 28 tertiary centers from January 2008 to January 2016. The basic eligibility criteria were that the patients were adults ( $\geq 18$ years) with histologically confirmed unresectable or metastatic adenocarcinoma of the distal esophagus, gastroesophageal junction, and stomach. The patients had to have received at least one 
cycle of polychemotherapy (two or more drugs) in the firstline treatment, and to have been followed up for at least 3 months with adequate clinical information; an objective tumor evaluation based on computed tomography was required, and laboratory data for this period had to be available. Subjects who died early are exempted from these requirements. Patients were excluded if the information needed to complete the database collection could not be obtained, or if they were still alive at the time of collection but did not give their written consent or exercised their right to rectify and revoke. The ethics review board at each of the participating institutions approved the study, and the procedures were in accordance with the Helsinki Declaration.

\section{Study design}

The Spanish National Cancer Registry is a non-interventionist cancer registry. HER2 testing and trastuzumab or chemotherapy regimens were implemented or not, depending on each center's clinical practice. Data were collected through the centralized web platform: http:// www.agamenonstudy.com/. This software tool has filters to ensure data consistency. In addition, an independent researcher monitored data accuracy remotely, validating information and locking data for cases when they were completed.

\section{Variables}

HER2 overexpression/amplification was tested according to clinical practice protocols at each center by means of IHC or FISH [12]. All patients with biopsy scores of IHC $3+$ were eligible for trastuzumab, as well as cases with biopsy scores of IHC $2+$ and a positive FISH amplification result. When the HER2 status was not determined according to the usual practice, researchers were not requested to obtain a retrospective assessment. Hospitals were classified according to the proportion of patients who were not tested for HER2, and divided by quartiles into four groups of approximately the same size, representing universal, high, medium, and low HER2 testing. Reasons for not ordering HER2 testing were recorded. The primary outcome measure was OS, calculated from the beginning of the first cycle of chemotherapy.

\section{Statistics}

Survival curves were estimated using the Kaplan-Meier method. The Wilcoxon test was applied to compare the survival functions. The effect of trastuzumab was assessed using stratified Cox proportional hazards $(\mathrm{PH})$ regression. When building this model, we analyzed 13 covariates using univariate statistical method screening; variables univariately predictive of survival $(P<0.1)$ were entered into the Cox PH regression. We excluded the covariate HER2 status to avoid multicollinearity with trastuzumab therapy. This analysis was stratified by year of diagnosis and controlled for other confounding factors. The PH assumption was determined using Schoenfeld residuals. Sensitivity analyses were performed according to the reason for not ordering HER2 testing. All $P$ values are two-tailed. The STATA statistical package v14 (StataCorp, College Station, TX, USA) was used throughout.

\section{Results}

\section{HER2 determination and use of trastuzumab}

The registry contained 1615 patients, 1170 of whom met the eligibility criteria (Fig. 1). Patient baseline characteristics are shown in Table 1. Overall, HER2 was not tested in $22.9 \%$ of the patients in the registry $(N=268)$. The rate of HER 2 testing increased over time, from $58.3 \%$ in 2008 to $92.9 \%$ in 2016 (Fig. 2). Alleged reasons for not determining HER2 in this registry were as follows: HER2 testing (or the use of trastuzumab) was not formalized as standard for AGC in this institution in $74.3 \%$, date of firstline therapy prior to 2010 in $8.3 \%$, unknown in $6.4 \%$, HER2 status tested for second-line therapy in $3.7 \%$, insufficient tissue sampling in $3.7 \%$, chemotherapy initiation deemed urgent, but no subsequent HER2 assessment due to deterioration or premature death in $3.3 \%$, and subjects were not candidates to receive trastuzumab therapy (e.g., elderly or severe heart disease) in $0.3 \%$.

Of the 908 tested tumors, HER2 was positive in 194 of them $(21.3 \%)$ (IHC $3+$ in 123 , IHC $2+/$ FISH+ in 71$)$. Trastuzumab was administered combined with first-line chemotherapy to 165 of these individuals. Two of these tumors, initially considered HER2-positive and treated with trastuzumab, were retested due to early progression, and finally both were classified as HER2-negative. In this series, trastuzumab associated with first-line chemotherapy was first administered in 2008. Since then, the percentage of patients with HER2-positive tumors who have received first-line trastuzumab has increased year after year (Fig. 2). The turning point occurred in 2011 when $90 \%$ of subjects with HER2-positive tumors received trastuzumab in combination with the first-line treatment.

HER2-positive patients treated with trastuzumab exhibited better prognosis than HER2-negative patients. Median OS was 14.6 months (CI 95\%, 11.1-18.1) in trastuzumab-treated patients as opposed to 9.7 months (CI $95 \%, 9.1-10.3$ ) in HER2-negative patients, $P<0.001$. In contrast, 31 patients with HER2-positive AGC did not 
Fig. 1 Flowchart of the study. Asterisk: note that the categories were not mutually exclusive
Patients assessed for eligibility $(n=1615)$

Not meeting inclusion criteria $(n=253)^{*}$

- Did not receive any chemotherapy $(n=169)$

- Unfit for combination chemotherapy $(n=233)$

Elderly $(n=37)$

Comorbidities ( $n=43$ )

Poor performance status $(n=129)$

Patient refusal $(n=8)$

Other $(n=16)$

- Less than 6 months since adjuvant therapy $(n=22)$

- Other metastatic primary tumor $(n=10)$

- Clinical trial $(n=10)$

- Previous chemotherapy for advanced disease $(n=22)$

- Declined to participate $(n=11)$

- Missing values $(n=90)$

- Lost to follow up $(n=13)$

- Insufficient (<3 months) follow-up $(n=78)$

Eligible patients $(n=1170)$

receive trastuzumab with first-line chemotherapy (see Table S1 in the Electronic supplementary material, ESM). Reasons for not administering first-line trastuzumab in these 31 HER2-positive cases were as follows: initiation of first-line therapy before 2010 with HER2 being tested for successive lines $(N=11)$; contraindication due to chronic heart disease $(N=5)$; still not considered standard by the treating physician $(N=7)$; and trastuzumab administration with the second-line treatment $(N=8)$. Despite the small subgroup $(N=31)$, the median OS in HER2-positive patients who did not receive first-line trastuzumab was 14.9 months (CI $95 \%$, 7.7-22.1). Remarkably, 19 of these 31 patients received trastuzumab with the second- or thirdline therapy.

\section{Impact of HER2 testing on outcomes}

In the univariate analysis, a positive effect on OS was observed for the IHC 2+/FISH+ subgroup, with a stratified HR of 0.71 (CI $95 \%, 0.53-0.97$ ), $P=0.032$, as well as for patients with IHC $3+$ status, with a stratified HR of 0.57 (CI $95 \%, 0.45-0.73), P<0.0001$. In contrast, patients in whom the HER2 testing was not performed had a worse OS compared to the rest of the series: they had a stratified HR of 1.226 (CI $95 \%, 1.035-1.451$ ), $P=0.018$, considering a HER2-negative status as the reference (see Table 2). However, this effect disappeared in the sensitivity analyses when subjects who died within 30 days of chemotherapy or for whom trastuzumab was contraindicated (e.g., the elderly or those with severe heart disease) were excluded.

Patients treated in the 4-year period 2012-2015 had a longer median OS, 10.4 months (CI $95 \%$, 9.64-11.24), as compared to patients treated during 2008-2011, with a median OS of 9.2 months (95\% CI, 8.2-10.2), $P=0.021$. Otherwise, patients treated at institutions with increasing proportions of tumors that were not tested for HER2 showed a significant increase in risk of death, with a stratified HR of 1.005 (CI 95\%, 1.003-1.007), $P<0.0001$. This effect remained significant after excluding either those subjects with trastuzumab contraindication, subjects with early mortality (median OS $<30$ days), or those who were treated before 2011.

Table S2 in the ESM displays the proportion of the patients who were not tested for HER2 at each institution 
Table 1 Baseline characteristics $(n=1170)$

\begin{tabular}{|c|c|}
\hline Characteristic & $N(\%)$ \\
\hline Gender, male & $805(68.7 \%)$ \\
\hline Age, median, range & $64(21-86)$ \\
\hline \multicolumn{2}{|l|}{ Primary tumor site } \\
\hline Distal esophagus & $69(5.9 \%)$ \\
\hline Gastroesophageal junction & $127(10.8 \%)$ \\
\hline Cardia & $126(10.8 \%)$ \\
\hline Body & $403(34.4 \%)$ \\
\hline Antrum & $258(22 \%)$ \\
\hline Pylorus & $32(2.7 \%)$ \\
\hline Fundus & $68(5.8 \%)$ \\
\hline Gastric stump/remnant & $9(0.8 \%)$ \\
\hline Multiple & $53(4.5 \%)$ \\
\hline Not available & $18(1.5 \%)$ \\
\hline \multicolumn{2}{|l|}{ Histological grade } \\
\hline 1 & $115(9.8 \%)$ \\
\hline 2 & $342(29.2 \%)$ \\
\hline 3 & $471(40.2 \%)$ \\
\hline No available & $235(20.2 \%)$ \\
\hline \multicolumn{2}{|l|}{ Lauren classification } \\
\hline Intestinal & $574(49 \%)$ \\
\hline Diffuse & $383(32.7 \%)$ \\
\hline Mixed & $62(5.3 \%)$ \\
\hline Not available/not classifiable & $144(12.3 \%)$ \\
\hline \multicolumn{2}{|l|}{ HER2 } \\
\hline Negative $(0+, 1+, 2+$ and FISH -$)$ & $708(60.5 \%)$ \\
\hline Positive $(3+)$ & $123(10.5 \%)$ \\
\hline Positive $(2+$ and FISH +$)$ & $71(6.1 \%)$ \\
\hline Not available & $268(22.9 \%)$ \\
\hline \multicolumn{2}{|l|}{ Signet ring cells } \\
\hline No & $695(59.3 \%)$ \\
\hline$<50 \%$ & $92(7.8 \%)$ \\
\hline$\geq 50 \%$ & $112(9.6 \%)$ \\
\hline Present with unknown percentage & $143(12.2 \%)$ \\
\hline Not available & $121(10.3 \%)$ \\
\hline \multicolumn{2}{|l|}{ Metastatic sites } \\
\hline Liver & $441(37.6 \%)$ \\
\hline Lung & $143(12.2 \%)$ \\
\hline Bone & $111(9.5 \%)$ \\
\hline Peritoneum & $529(45.1 \%)$ \\
\hline Ascites & $286(24.4 \%)$ \\
\hline \multicolumn{2}{|l|}{ First-line chemotherapy } \\
\hline Doublet & $759(64.8 \%)$ \\
\hline Triplet & $413(35.2 \%)$ \\
\hline \multicolumn{2}{|l|}{ Chemotherapy regimens } \\
\hline Oxaliplatin-based & $405(34.6 \%)$ \\
\hline Anthracycline-based & $286(24.4 \%)$ \\
\hline Cisplatin-based & $219(18.7 \%)$ \\
\hline Docetaxel-based & $168(14.3 \%)$ \\
\hline
\end{tabular}

Table 1 continued

\begin{tabular}{ll}
\hline Characteristic & $N(\%)$ \\
\hline Irinotecan-based & $20(1.7 \%)$ \\
Other & $48(4.1 \%)$ \\
Not available & $26(2.2 \%)$ \\
Trastuzumab in first-line & $165(14.1 \%)$ \\
ECOG PS & \\
0 & $260(22.2 \%)$ \\
1 & $750(64 \%)$ \\
2 & $153(13.1 \%)$ \\
3 & $8(0.7 \%)$ \\
4 & $1(0.1 \%)$ \\
Year of first-line therapy & \\
$2008-2009$ & $138(11.8 \%)$ \\
$2010-2011$ & $191(16.3 \%)$ \\
$2012-2013$ & $390(33.3 \%)$ \\
$2014+$ & $451(38.5 \%)$ \\
\hline
\end{tabular}

(encoded). This shows that the rate of non-testing at institutions was not homogeneous. The centers were therefore classified by quartiles based on the proportion of patients with missing HER2 data, which divided the set of measurements into four approximately equal parts $\left(Q_{1}-Q_{4}\right.$, see Table 3).

Kaplan-Meier curves and survival times for $Q_{1}-Q_{4}$ are presented in Fig. 3 and Table 3. The median OS progressively decreased for quartiles $Q_{1}$ to $Q_{4}: 10.67$ months (95\% CI, 9.62-11.72) for $Q_{1}, 10.28$ months $(95 \% \mathrm{CI}$, 8.91-11.65) for $Q_{2}, 9.98$ months (95\% CI, 8.43-11.54) for $Q_{3}$, and 8.57 months (95\% CI, 7.44-10.67) for $Q_{4}$. The differences in median OS between the fourth quartile $\left(Q_{4}\right.$, missing HER2 data $>30 \%)$ and the other quartiles $\left(Q_{1}-\right.$ $Q_{3}$ ) were significant. Pairwise comparisons of median OS were as follows: $Q_{1}$ vs. $Q_{2}, P=0.642 ; Q_{1}$ vs. $Q_{3}$, $P=0.381 ; Q_{1}$ vs. $Q_{4}, P=0.001 ; Q_{2}$ vs. $Q_{3}, P=0.190 ;$ $Q_{2}$ vs. $Q_{4}, P=0.001$; and $Q_{3}$ vs. $Q_{4}, P=0.034$. Differences among institutions remained significant when patients treated before 2011 were excluded.

In the stratified $\mathrm{Cox} \mathrm{PH}$ regression, the proportion of the patients who were not tested for HER2 at the institutions was significantly associated with adverse outcomes (Table 4). Each $1 \%$ increase in the patients with HER2 missing data at each hospital induced a significant approximately $0.3 \%$ increase in the risk of death during this period of observation: HR, 1.0035 (CI $95 \%$, 1.0014-1.0057), $P=0.0019$, with the latter being specifically stratified by year of diagnosis. For example, a hypothetical institution that tested HER 2 in only $65 \%$ of AGC patients would have an approximately $10 \%$ increase in the risk of death in comparison with a center in which every patient is tested. 


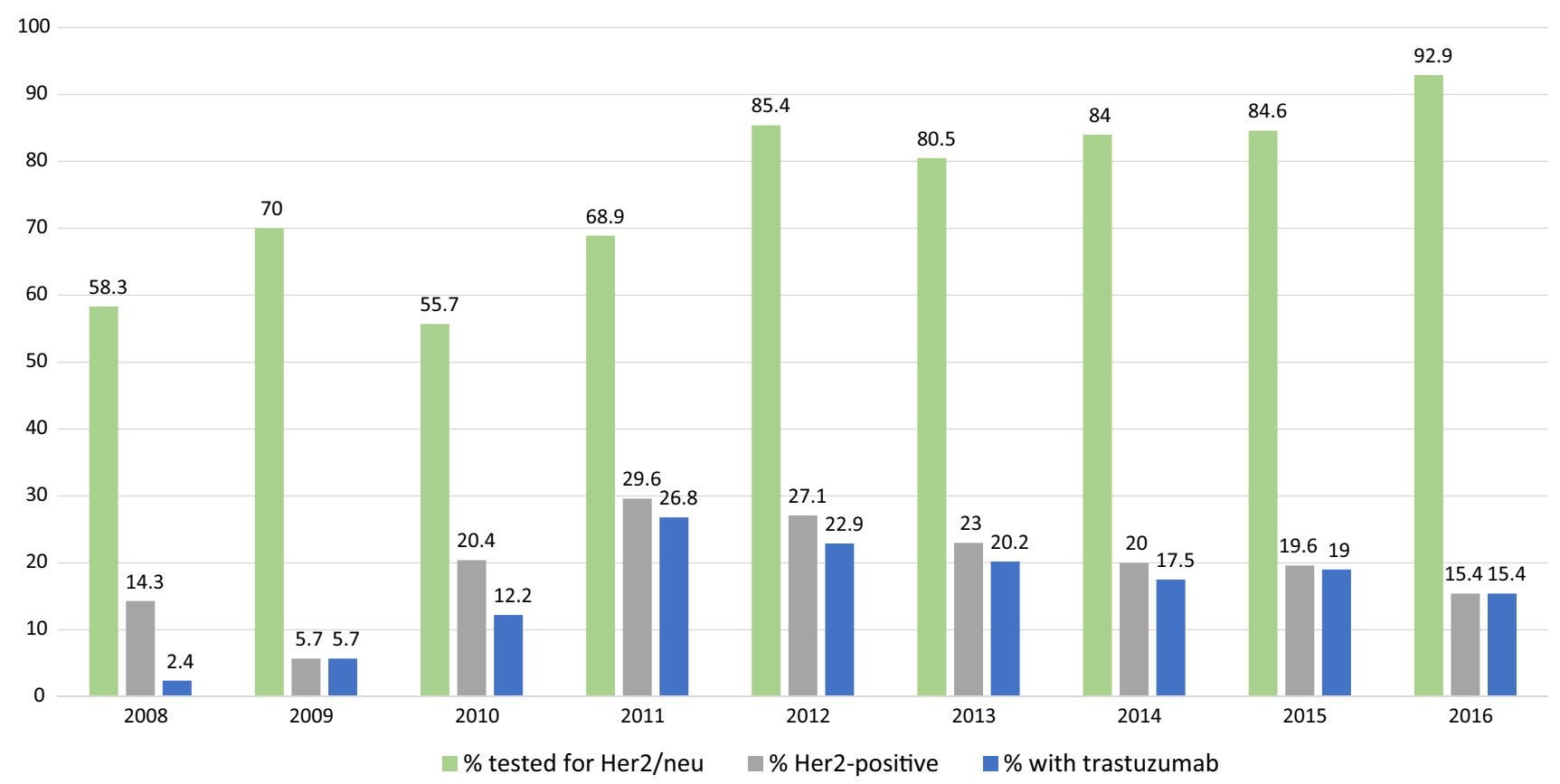

Fig. 2 Patients tested for HER2 and treated with trastuzumab each year. Percentages of HER2-positive patients (IHC $3+$ or IHC $2+$ and FISH+) and patients treated with trastuzumab with respect to the total number of patients tested for HER2 are shown

A secondary consequence of HER2 testing in this series is the change in the pattern of chemotherapy use, with a gradual reduction in the use of triplet chemotherapy in parallel with increased HER2 testing. Thus, chemotherapy with three cytotoxic agents was used in $13 \%$ of the HER2-positive (IHC +3 ) patients and for $4.2 \%$ of the HER2-positive (IHC +2 / FISH+) tumors, whereas $40 \%$ of the HER2-negative patients and those whose HER2 status was undetermined were treated with triple chemotherapy $(P<0.0001)$.

\section{Discussion}

Gastric cancer is a common malignancy associated with poor prognosis. It is therefore imperative that new, more effective treatment strategies should be developed and quickly incorporated into daily clinical practice. An analysis of genomic aberrations has shown that signaling pathways for genes such as HER2, vascular endothelial growth factor (VEGF), phosphatidylinositol-4,5-bisphosphate 3-kinase/AKT/mammalian target of rapamycin (PI3K/AKT/mTOR), and hepatocyte growth factor and its receptor $\mathrm{MET}$ (HGF/MET) are often altered in gastric cancer [2]. Inhibiting agents that target these molecular anomalies are a very promising therapeutic option [14].

According to the ToGA study data, trastuzumab combined with chemotherapy unequivocally increases survival in HER2-positive AGC [10]. However, the literature is less clear about how these advances in knowledge trickle down into clinical practice in terms of the implementation of HER2 testing, how to overcome the barriers that impede the use of this new drug, and how this will ultimately serve to bring the benefits observed in the trial into daily clinical practice.

In 2015, we began a clinical practice registry covering 28 centers to detect areas for improvement in the management of AGC. The registry suggests that HER2 was relatively rapidly introduced as up to 11 centers began HER testing in the biennium 2008-2009. Consequently, $63 \%$ of the cancers were tested in that time period. Because the registry includes relatively few cases from these years and brings together the main Spanish reference centers but not smaller institutions, it is difficult to know whether this truly represents clinical practice in the country during 2008-2009. Nonetheless, this result is striking, as little information had been published about the effectiveness of trastuzumab at that time [15, 16]. In addition, trastuzumab had not been approved during that period; hence, in most of these cases, it was not used as first-line therapy.

The ToGA study was published in August 2010 [10], and had a strong impact on clinical management. A few months following ToGA study publication [10], trastuzumab was commonly used with first-line chemotherapy in almost all HER2-positive tumors. Since then, the percentage of tumors tested has increased year after year, although HER2 testing did not reach $85 \%$ until 2012, and universal testing was probably not achieved in Spain until very 
Table 2 Overall survival by subgroups (univariate analyses)

\begin{tabular}{|c|c|c|c|c|c|}
\hline & Median OS (months) & Survival rate at 12 months & $95 \% \mathrm{CI}$ & HR & $P$ value \\
\hline All patients & 10.0 & 42.1 & $38.9-45.2$ & - & - \\
\hline Proportion of patients tested for HER2 at institutions* & - & - & - & 1.005 & $<0.0001$ \\
\hline HER2 status of the patient & & & & & $<0.0001$ \\
\hline HER2-negative & 10.0 & 41.9 & $37.9-45.8$ & Ref. & - \\
\hline HER2-positive & 14.6 & 57.5 & $50.2-64.7$ & 0.622 & $<0.0001$ \\
\hline HER2-missing & 8.1 & 31.3 & $25.2-37.3$ & 1.226 & 0.018 \\
\hline Lauren classification & & & & & $<0.0001$ \\
\hline Non-diffuse & 11.3 & 47.2 & $42.8-51.5$ & Ref. & Ref. \\
\hline Diffuse tumors & 8.8 & 37.8 & $32.9-42.7$ & 1.316 & $<0.0001$ \\
\hline Unknown & 8.3 & 34.8 & $26.5-43.0$ & 1.402 & 0.001 \\
\hline Histological grade & & & & & $<0.0001$ \\
\hline G1 & 16.0 & 63.8 & $54.5-73.0$ & Ref. & - \\
\hline G2 & 9.5 & 39.9 & $34.4-45.3$ & 1.990 & $<0.0001$ \\
\hline G3 & 9.7 & 40.3 & $35.5-45.6$ & 1.909 & $<0.0001$ \\
\hline NR & 9.5 & 30.8 & $24.1-37.8$ & 2.041 & $<0.0001$ \\
\hline \multicolumn{6}{|l|}{ Trastuzumab in first-line treatment } \\
\hline No & 9.7 & 39.8 & $36.0-43.5$ & Ref. & $<0.0001$ \\
\hline Yes & 14.6 & 55.9 & $47.8-63.9$ & 0.599 & \\
\hline \multicolumn{6}{|l|}{ Signet ring cells } \\
\hline No & 10.5 & 44.2 & $40.4-47.9$ & Ref. & 0.025 \\
\hline Yes & 8.8 & 37.2 & $31.7-42.6$ & 1.185 & \\
\hline Chemotherapy & & & & & 0.068 \\
\hline Doublet & 9.7 & 40.4 & $36.6-44.1$ & Ref. & \\
\hline Triplet & 10.7 & 45.2 & $40.1-50.2$ & 0.876 & \\
\hline Age, years* & - & - & - & 0.998 & 0.611 \\
\hline \multicolumn{6}{|l|}{ ECOG PS } \\
\hline $0-1$ & 10.8 & 46.1 & $42.7-49.4$ & Ref. & $<0.0001$ \\
\hline$\geq 2$ & 5.4 & 18.1 & $11.8-24.3$ & 2.204 & \\
\hline \multicolumn{6}{|l|}{ Bone metastases } \\
\hline No & 10.5 & 44.5 & $41.3-47.6$ & Ref. & $<0.0001$ \\
\hline Yes & 6.8 & 21.6 & $13.5-29.6$ & 1.752 & \\
\hline \multicolumn{6}{|l|}{ Ascites } \\
\hline No & 10.6 & 45.0 & $41.4-48.5$ & Ref. & $<0.0001$ \\
\hline Yes & 8.2 & 33.1 & $27.2-38.9$ & 1.478 & \\
\hline \multicolumn{6}{|l|}{ Lung metastases } \\
\hline No & 10.0 & 42.1 & $38.7-45.4$ & Ref. & 0.972 \\
\hline Yes & 10.2 & 42.5 & $33.8-51.1$ & 1.004 & \\
\hline \multicolumn{6}{|l|}{ Peritoneal metastases } \\
\hline No & 10.8 & 45.2 & $41.0-49.3$ & Ref. & 0.004 \\
\hline Yes & 9.1 & 38.5 & $33.9-43.0$ & 1.215 & \\
\hline
\end{tabular}

OS overall survival, $C I$ confidence interval, $H R$ hazard ratio, ECOG PS Eastern Cooperative Group Performance Status, Ref reference value, NR not reported

$P$ values were calculated from univariate Cox PH regression. Survival rates were estimated from the life tables in the Kaplan-Meier method

recently, despite the 2011 national consensus [12]. Nevertheless, we noticed that the introduction of HER2 testing did not occur synchronously nor homogeneously throughout the country. This is possibly due to the decentralization of the national health system in Spain, and it appears to have had a noticeable clinical impact at different regional institutions. To analyze this issue, each of the hospitals included in this registry was classified on the basis of the 
Table 3 Median overall survival (OS) and hazard ratio (HR) based on missing HER2 data quartiles

\begin{tabular}{llllll}
\hline & $N(\%)$ & Number of centers & Boundary for missing HER2 categories & Median OS, months (CI 95 \%) & HR (CI 95 \%) \\
\hline$Q_{1}$ & $416(35.6 \%)$ & 13 & Universal $(0 \%)$ & $10.67(9.62-11.72)$ & Reference \\
$Q_{2}$ & $188(16.1 \%)$ & 4 & High $(1-3 \%)$ & $10.28(8.91-11.65)$ & $1.04(0.86-1.27)$ \\
$Q_{3}$ & $251(21.5 \%)$ & 6 & Medium $(4-30 \%)$ & $9.98(8.43-11.54)$ & $1.13(0.95-1.35)$ \\
$Q_{4}$ & $315(26.9 \%)$ & 5 & Low $(>30 \%)$ & $8.57(7.44-10.67)$ & $1.34(1.13-1.59)$ \\
\hline
\end{tabular}

$H R$ hazard ratio, $O S$ overall survival

Pairwise comparisons of median overall survival (OS): $Q_{1}$ vs. $Q_{2}(P=0.642), Q_{1}$ vs. $Q_{3}(P=0.381), Q_{1}$ vs. $Q_{4}(P=0.001), Q_{2}$ vs. $Q_{3}$ $(P=0.190), Q_{2}$ vs. $Q_{4}(P=0.001), Q_{3}$ vs. $Q_{4}(P=0.034)$ (Wilcoxon test)

Table 4 Stratified Cox proportional hazards model for overall survival (OS)

\begin{tabular}{|c|c|c|c|c|c|}
\hline Covariate & Estimate & SE & HR & $95 \% \mathrm{CI}$ & $P$ value \\
\hline Proportion of patients not tested for HER2 at institutions* & 0.003520 & 0.001134 & 1.0035 & $1.0013-1.0057$ & 0.0019 \\
\hline Trastuzumab in first-line treatment & -0.4112 & 0.1099 & 0.6629 & $0.5351-0.8213$ & 0.0002 \\
\hline \multicolumn{6}{|l|}{ Lauren classification } \\
\hline Intestinal & Ref. & Ref. & Ref. & Ref. & Ref. \\
\hline Diffuse & 0.08794 & 0.09931 & 1.0919 & $0.8997-1.3252$ & 0.3759 \\
\hline Mixed & 0.07465 & 0.1568 & 1.0775 & $0.7937-1.4628$ & 0.6340 \\
\hline Not available/not classifiable & 0.1631 & 0.1149 & 1.1771 & $0.9408-1.4728$ & 0.1559 \\
\hline \multicolumn{6}{|l|}{ Histological grade } \\
\hline 1 & Ref. & Ref. & Ref. & Ref. & Ref. \\
\hline 2 & 0.5183 & 0.1335 & 1.6792 & $1.2943-2.1786$ & 0.0001 \\
\hline 3 & 0.3419 & 0.1382 & 1.4076 & $1.0750-1.8432$ & 0.0134 \\
\hline No available & 0.3704 & 0.1486 & 1.4483 & $1.0840-1.9352$ & 0.0127 \\
\hline Signet ring cells, presence & -0.01817 & 0.09391 & 0.9820 & $0.8177-1.1793$ & 0.8465 \\
\hline Chemotherapy, triplet & -0.2303 & 0.07549 & 0.7943 & $0.6856-0.9202$ & 0.0023 \\
\hline ECOG PS, $\geq 2$ & 0.6784 & 0.09548 & 1.9708 & $1.6360-2.3741$ & $<0.0001$ \\
\hline Peritoneal metastases & 0.08787 & 0.08164 & 1.0919 & $0.9312-1.2803$ & 0.2818 \\
\hline Ascites & 0.2396 & 0.09183 & 1.2708 & $1.0624-1.5200$ & 0.0091 \\
\hline Bone metastases & 0.5953 & 0.1087 & 1.8137 & $1.4672-2.2419$ & $<0.0001$ \\
\hline
\end{tabular}

$C I$ confidence interval, $S E$ standard error (of estimate), $H R$ hazard ratio, Ref. reference value, ECOG PS Eastern Cooperative Oncology Group Performance Status

* The percentage of patients not tested for HER2 at institutions and age were analyzed as continuous variables. Cox regression was stratified by the year of first-line therapy. Test of proportional hazards assumption $\chi^{2}=18.18, d f=11, P=0.077$

rate of missing HER2 data. Based on the four quartiles of this distribution, the centers were categorized into four groups: universal, high, medium, and low HER2 testing. Generally, prognosis worsened from the universal to the high to the medium and then to the low HER2 testing group, such that a statistically significant difference in median OS of 2.1 months was observed between the universal and low HER2 testing groups.

Two main ideas emerge from this analysis. First, many patients with AGC were missing out on a crucial genetic test to determine whether they would benefit from trastuzumab. Second, the favorable effect of widespread HER2 testing does not seem to be limited to patient selection. This implies that HER2-negative patients might also have benefited in some way from the proper implementation of
HER2 testing at their center. A plausible interpretation of these data is that the percentage of HER2-tested patients behaves as a quality-of-care indicator at these centers. It is tempting to speculate that determining HER2 status in clinical practice is a challenge for the institution, which forced multidisciplinary teams to coordinate, improving the quality of care in many different ways beyond assisting in patient selection for anti-HER2 therapy [12]. As such, the policy of universal genetic testing seems to behave as a quality-of-care indicator. However, it is also necessary to keep in mind that not determining HER2 due to poor clinical status or trastuzumab contraindication can be regarded as good practice. On the other hand, centers that determined HER2 less frequently tended to supplement this practice with the more frequent use of triplet instead of 
Fig. 3 Kaplan-Meier curves for OS by percentile. Centers are classified by quartile, based on the proportion of patients with a missing HER2 determination: $Q_{1}=0 \%$, $Q_{2}=1-3 \%, Q_{3}=4-30 \%$, and $Q_{4}>30 \%$. Median OS and HR based on missing HER2 data quartiles are presented in Table 3

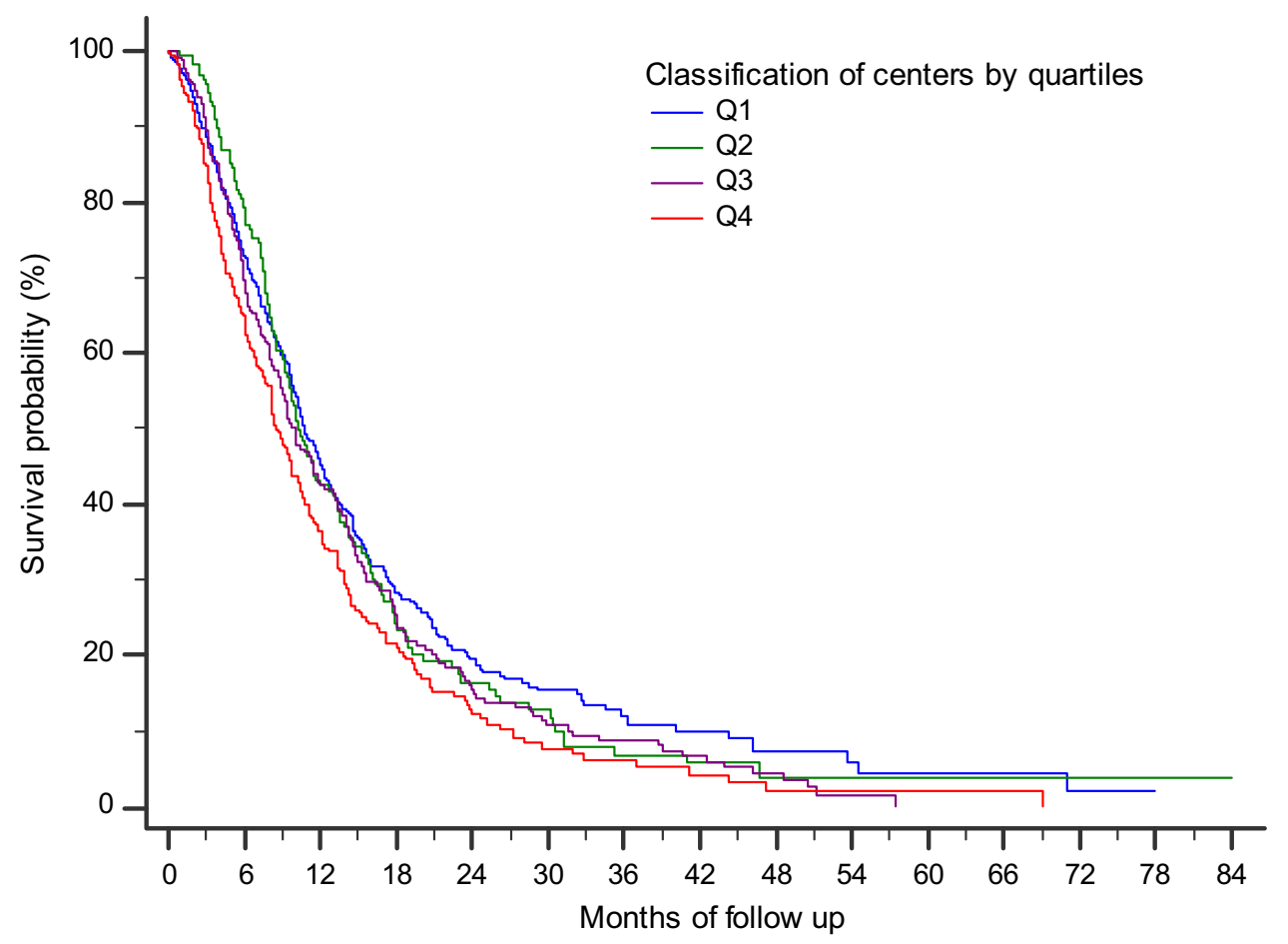

doublet chemotherapy (e.g., docetaxel-based regimens). A multivariate analysis of the cases included in our registry illustrates that this practice can be beneficial, but it does not completely palliate the negative effect of leaving HER2 untested.

Our study has several limitations inherent to retrospective registries, especially those dealing with complex spatial and temporal patterns. First, we cannot exclude the possibility that other reasons that were not taken into consideration may have confounded patient prognosis. One of the most obvious concerns was that early mortality interferes competitively with HER2 testing. To control this potential bias, our analysis was stratified based on patient demise prior to 30 days. Second, as with all observational records, it is impossible to guarantee that all confounding factors, particularly time-dependent ones, have been taken into account. Third, the groups with low and intermediate levels of HER2 testing include only a few hospitals ( 7 and 5 of 28 centers, respectively), and it is possible that local, nonHER2 testing practices at these institutions may have influenced patient prognosis.

Despite these limitations and caveats, we consider that our findings are consistent with the fact that early introduction of HER2 testing and trastuzumab therapy were beneficial in the patients included in this registry. In contrast, centers that took longer to implement HER2 testing exhibited worse clinical outcomes. We believe that these data could be extrapolated to other biomarkers used to select patients for personalized oncology therapies. Centers should strive to incorporate these diagnostic improvements as soon as there is evidence of the benefit and cost-effectiveness of this procedure. Reviewed local and global guidelines on HER2 testing should be used to achieve this goal in a timely manner [12, 17-19].

Acknowledgments Priscilla Chase Duran for editing the manuscript. Natalia Cateriano, Miguel Vaquero, and IRICOM S.A. for supporting the registry website.

\section{Compliance with ethical standards}

Funding None to declare; this is an academic study. The registry was supported by the authors themselves.

Conflict of interest The authors declare that they have no conflict of interest.

Research involving human participants All procedures followed were in accordance with the ethical standards of the responsible committee on human experimentation (institutional and national) and with the Helsinki Declaration of 1964 and later versions. Informed consent or a substitute for it was obtained from all patients before they were included in the study.

\section{References}

1. Dulak AM, Schumacher SE, van Lieshout J, Imamura Y, Fox C, Shim B, et al. Gastrointestinal adenocarcinomas of the esophagus, stomach, and colon exhibit distinct patterns of genome instability and oncogenesis. Cancer Res AACR. 2012;72:4383-93. 
2. Deng N, Goh LK, Wang H, Das K, Tao J, Tan IB, et al. A comprehensive survey of genomic alterations in gastric cancer reveals systematic patterns of molecular exclusivity and co-occurrence among distinct therapeutic targets. Gut. 2012;61:673-84.

3. CGAR Network. Comprehensive molecular characterization of gastric adenocarcinoma. Nature. 2014;513:202-9.

4. Sakai K, Mori S, Kawamoto T, Taniguchi S, Kobori O, Morioka $\mathrm{Y}$, et al. Expression of epidermal growth factor receptors on normal human gastric epithelia and gastric carcinomas. J Natl Cancer Inst. 1986;77:1047-52. Available from: http://www.ncbi. nlm.nih.gov/pubmed/3464796.

5. Tokunaga A, Onda M, Okuda T, Teramoto T, Fujita I, Mizutani T, et al. Clinical significance of epidermal growth factor (EGF), EGF receptor, and c-erbB-2 in human gastric cancer. Cancer. 1995;75:1418-25.

6. Ishida T, Tsujisaki M, Hanzawa Y, Hirakawa T, Hinoda Y, Imai $\mathrm{K}$, et al. Significance of erbB-2 gene product as a target molecule for cancer therapy. Scand J Immunol. 1994;39:459-66.

7. Lordick F, Bang YJ, Kang YK, Reyes DO, Manikhas GM, Shen L, et al. 3541 POSTER HER2-positive advanced gastric cancer: similar HER2-positivity levels to breast cancer. Eur J Cancer Suppl. 2007;5:272.

8. Gravalos C, Jimeno A. HER2 in gastric cancer: a new prognostic factor and a novel therapeutic target. Ann Oncol Eur Soc Med. 2008;19:1523-9.

9. Cortés-Funes H, Rivera F, Alés I, Márquez A, Velasco A, Colomer R, et al. Phase II of trastuzumab and cisplatin in patients (pts) with advanced gastric cancer (AGC) with HER2/neu overexpression/amplification. ASCO Annu Meet Proc. 2007;25:4613.

10. Bang YJ, Van Cutsem E, Feyereislova A, Chung HC, Shen L, Sawaki A, et al. Trastuzumab in combination with chemotherapy versus chemotherapy alone for treatment of HER2-positive advanced gastric or gastro-oesophageal junction cancer (ToGA): a phase 3, open-label, randomised controlled trial. Lancet. 2010;376:687-97.
11. Satoh T, Bang YJ, Gotovkin EA, Hamamoto Y, Kang YK, Moiseyenko VM, et al. Quality of life in the Trastuzumab for Gastric Cancer trial. Oncologist. 2014;19:712-9.

12. Gómez-Martín C, Concha Á, Corominas JM, García-Caballero T, García-García E, Iglesias M, et al. Consensus of the Spanish Society of Medical Oncology (SEOM) and Spanish Society of Pathology (SEAP) for HER2 testing in gastric carcinoma. Clin Transl Oncol. 2011;13:636-51.

13. Dickman PW, Sloggett A, Hills M, Hakulinen T. Regression models for relative survival. Stat Med. 2004;23:51-64.

14. Riquelme I, Saavedra K, Espinoza JA, Weber H, García P, Nervi $\mathrm{B}$, et al. Molecular classification of gastric cancer: towards a pathway-driven targeted therapy. Oncotarget. 2015;6:24750-79.

15. Nicholas G, Cripps C, Au HJ, Jonker D, Salim M, Bjarnason G, et al. Early results of a trial of trastuzumab, cisplatin, and docetaxel (TCD) for the treatment of metastatic gastric cancer overexpressing HER-2. Ann Oncol. 2006;17:ix316.

16. Rech J, Arnold D, Folprecht G, Hejna M, Hofmann M, Gramatzki M. A pilot study of trastuzumab monotherapy in patients who progressed while on chemotherapy for metastatic or locally advanced HER2-positive gastric cancer. Ann Oncol. 2006; 17:ix314.

17. Bartley AN, Christ J, Fitzgibbons PL, Hamilton SR, Kakar S, Shah MA, et al. Template for reporting results of HER2 (ERBB2) biomarker testing of specimens from patients with adenocarcinoma of the stomach or esophagogastric junction. Arch Pathol Lab Med. 2015;139:618-20. Available from: http://www.ncbi. nlm.nih.gov/pubmed/25275813.

18. Rüschoff J, Hanna W, Bilous M, Hofmann M, Osamura RY, Penault-Llorca F, et al. HER2 testing in gastric cancer: a practical approach. Mod Pathol. 2012;25:637-50.

19. Whitney-Miller C, Hicks DG. College of American Pathologists guidelines for reporting HER2 test results in gastric cancer. Arch Pathol Lab Med. 2015;139:709-10. Available from: http://www. ncbi.nlm.nih.gov/pubmed/25295581. 